\title{
Oral human Papillomavirus DNA detection in HIV-positive men: prevalence, predictors, and co-occurrence at anal site
}

Alessandra Vergori ${ }^{1}$, Anna Rosa Garbuglia ${ }^{2,5^{*}}$, Pierluca Piselli ${ }^{3}$, Franca Del Nonno ${ }^{4,5}$, Catia Sias ${ }^{2}$, Federico Lupi ${ }^{1}$, Daniele Lapa ${ }^{2}$, Andrea Baiocchini ${ }^{4}$, Claudia Cimaglia ${ }^{3}$, Marco Gentile ${ }^{1}$, Andrea Antinori ${ }^{1}$, Maria Capobianchi ${ }^{2}$ and Adriana Ammassari ${ }^{1}$

\begin{abstract}
Background: HIV-positive patients carry an increased risk of HPV infection and associated cancers. Therefore, prevalence and patterns of HPV infection at different anatomical sites, as well as theoretical protection of nonavalent vaccine should be investigated. Aim was to describe prevalence and predictors of oral HPV detection in HIV-positive men, with attention to nonavalent vaccine-targeted HPV types. Further, co-occurrence of HPV DNA at oral cavity and at anal site was assessed.

Methods: This cross-sectional, clinic-based study included 305 HIV-positive males (85.9\% MSM; median age 44.7 years; IQR: 37.4-51.0), consecutively observed within an anal cancer screening program, after written informed consent. Indication for anal screening was given by the HIV physician during routine clinic visit. Paired oral rinse and anal samples were processed for the all HPV genotypes with QIASYMPHONY and a PCR with MY09/MY11 primers for the L1 region.

Results: At the oral cavity, HPV DNA was detected in 64 patients (20.9\%), and in $28.1 \%$ of these cases multiple HPV infections were found. Prevalence of oral HPV was significantly lower than that observed at the anal site $(p<0.001)$, where HPV DNA was found in 199 cases $(85.2 \%)$. Oral HPV tended to be more frequent in patients with detectable anal HPV than in those without $(p=0.08)$. Out of 265 HPV DNApositive men regardless anatomic site, 59 cases (19.3\%) had detectable HPV at both sites, and 51 of these showed completely different HPV types. At least one nonavalent vaccine-targeted HPV type was found in $17 / 64(26.6 \%)$ of patients with oral and 199/260 (76.5\%) with anal infection. At multivariable analysis, factors associated with positive oral HPV were: CD4 cells $<200 / \mu \mathrm{L}$ (versus CD4 cells $>200 / \mu \mathrm{L}, p=0.005$ ) and $>5$ sexual partners in the previous 12 months (versus $0-1$ partner, $p=0.008$ ).

Conclusions: In this study on Italian HIV-positive men (predominantly MSM), oral HPV DNA was detected in approximately one fifth of tested subjects, but prevalence was significantly lower than that observed at anal site. Low CD4 cell count and increasing number of recent sexual partners significantly increased the odds of positive oral HPV. The absence of co-occurrence at the two anatomical sites may suggest different routes or timing of infection.
\end{abstract}

Keywords: HPV, HIV, Oral cavity, Anal site, Vaccine-targeted infections

\footnotetext{
* Correspondence: annarosa.garbuglia@inmi.it

2Laboratory of Virology, INMI "L.Spallanzani", Rome, Italy

${ }^{5}$ National Institute for Infectious Diseases, IRCCS L.Spallanzani, Via Portuense,

292, 00149 Rome, Italy

Full list of author information is available at the end of the article
}

(c) The Author(s). 2018 Open Access This article is distributed under the terms of the Creative Commons Attribution 4.0 International License (http://creativecommons.org/licenses/by/4.0/), which permits unrestricted use, distribution, and reproduction in any medium, provided you give appropriate credit to the original author(s) and the source, provide a link to the Creative Commons license, and indicate if changes were made. The Creative Commons Public Domain Dedication waiver (http://creativecommons.org/publicdomain/zero/1.0/) applies to the data made available in this article, unless otherwise stated. 


\section{Background}

In the current era of combination antiretroviral therapy (cART), tumours have become the leading cause of mortality in HIV-positive persons [1, 2]. Among non-AIDS cancers, HPV-associated tumours recently gained great attention because of multi-site distribution (genital tract, anus, head and neck), availability of screening procedures, and need of vaccine allocation strategies. HIVpositive persons experience a 3-fold higher standardized incidence of head and neck squamous cell carcinoma (HNSCC) than general population [3], and about $50 \%$ of oro-pharyngeal squamous cell carcinoma (OSCC) are HPV-related tumours, as found in a large retrospective study that investigated the prevalence of HPV infection in cancer tissues from more than 1600 patients diagnosed in the UK during the last decade [4]. Notably, HIV-positive persons were 2.1 times more likely to harbor HPV in the oral cavity in comparison to HIVnegative subjects [5]. Screening studies conducted in the HIV-infected population demonstrated an overall prevalence of HPV DNA in the oral cavity was between 20 and $45 \%$, with the finding of the oncogenic type HPV16 between 12 and $26 \%$ [6-9].

In a meta-analysis of 26 publications focusing on men having sex with men (MSM), pooled prevalence of any HPV at the oral cavity was $17.1 \%$ (95\% CI 7.3-26.8\%) in HIV-negative and $28.9 \%$ (95\% CI 19.1-38.7\%) in HIVpositive MSM [9]. Overall, factors predictive of oral HPV infection were: HIV infection, severe immunodepression and number of sexual partners $[9,10]$.

Concerning multi-site HPV infections, particularly MSM were at high risk of HPV co-occurrence at multiple anatomic sites, as shown in a study that found 59.1\% MSM with multi-site HPV occurrence (34.1\% and $22.7 \%$ at two and three sites respectively) [11]. Yet, detection of the same HPV types at the oral cavity and the anus can be as low as 5\% [7, 9, 12-14]. Yet, evaluation of HPV DNA co-occurrence at diverse anatomic sites should be evaluated in different geographical and clinical settings, because it may have impact on the clinical management of HIV-infected persons with regards to HPV detection and cancer screening strategies.

Vaccines to prevent HPV infection have been developed and are approved in some countries for immunization programs in adolescent females, as well as in boys and young men $[15,16]$. Currently, the U.S. Food and Drug Administration has approved the nonavalent vaccine (Gardasil $9^{\circ}$, Merck \& Co., Inc., Whitehouse Station, NJ) targeting HPV types $6,11,16,18,31,33,45,52,58$ for prevention of cervical, vulvar, vaginal and anal cancers and ano-genital warts [17-19]. Studies found the oncogenic type HPV16 in the oral cavity among 12 to $26 \%$ of cases [6-9], and prevalence of HPV types covered by the nonavalent vaccine in a study on 90 MSM was $77.8 \%$ in HIV-positive subjects, with HPV
58 and 16 mostly detected [11]. Since HPV vaccines have never been investigated for the prevention of OSCC, availability of more knowledge regarding the prevalence of nonavalent vaccine-targeted HPV types in HIV-infected patients with detectable HPV at the oral cavity would add some relevant information on the proportion of patients that in theory would have been protected if the vaccine had been administered before exposure.

In Italy at present not much is known about prevalence and predictors of oral HPV DNA detection in HIVinfected patients, and literature about co-occurrence of HPV infection in oral cavity and at anal site is scanty. For this reason, only few HIV/AIDS centres investigate presence of HPV in oral rinses and offer oral cancer screening to their patients. The aim of our study was to describe prevalence and predictors of oral HPV DNA detection in HIV-positive men attending an anal cancer screening program, with attention to vaccine-targeted HPV types. Further, we investigated co-occurrence of HPV DNA at the oral cavity and at the anal site.

\section{Methods \\ Study design and population}

This cross-sectional, clinic-based study was designed and conducted at the National Institute for Infectious for Disease, Rome, Italy, according to the Helsinki declaration and approved by the Institutional Review Board (n.42-18/6/2013).

All HIV-positive men, aged 18 years or older, consecutively observed within an anal cancer screening program between February 2015 and June 2016 provided written informed consent. The indication for anal screening was given based on current European AIDS Clinical Society (EACS) guidelines [20] by the HIV physician during routine clinic visit. Patients with history of anal warts or of pre-cancerous lesions were not excluded.

A questionnaire on health behaviours was created ad hoc and interviewer-administered. Questions focused on the following information: demographics, tobacco and alcohol use (more than 3 units per day), previous history of anal/genital condylomatosis, number of sexual partners in lifetime and in the previous 12 months, oral and anal sexual intercourse in lifetime and in the previous 12 months.

Clinical charts were used to abstract presence and type of current cART, as well as viroimmunological parameters (CD4 cell count and HIV RNA needed to be determined within 3 months of samples collection).

\section{HPV DNA testing}

At the same time of anal cancer screening, participants provided a sample of oral rinse obtained by swishing and gargling in the oral cavity $10-15 \mathrm{ml}$ of bottle mineral water (Fonte Tullia) for 20-30 s and spitting into a sterile specimen cup. One aliquot $(5-7 \mathrm{ml})$ was sent to the 
Laboratory of Pathology for cytological evaluation, while the second was centrifuged for $15 \mathrm{~min}$ at $3000 \mathrm{~g}$. Cell pellet was washed with sterile phosphate buffered saline and re-suspended in RPMI medium. Nucleic acids were extracted by automated QIASYMPHONY purification system (QIAGEN, Hilden, Germany). QuantiFast Pathogen Internal Control (QIAGEN, Hilden, Germany), was used to check presence of inhibiting factors. Furthermore, to ensure specimen adequacy, i.e. the presence of DNA, the samples were tested by amplification of a $110 \mathrm{bp}$ fragment of the human $\beta$-globin gene using $\mathrm{PCO} / \mathrm{PCO} 4$ primers [21] followed by analysis in $2 \%$ agarose gel. Samples positive for $\beta$-globin were considered evaluable.

For HPV DNA testing, a PCR for the L1 region was used. Products were analysed by electrophoresis in $1.7 \%$ agarose gel and stained with ethidium bromide, as previously described [22]. HPV-positive samples by MY09/11 PCR were typed using Genomica CLART assay. Samples, that could not be typed with the CLART assay, were directly sequenced using a Big Dye Terminator Cycle Sequencing Kit (Applied Biosystems, Ca). Sequence alignments were obtained using returned results from GeneBank's on-line BLAST server (http//https://blast.ncbi.nlm.nih.gov/Blast.cgi, nih.gov/BLAST) [23]. A similarity above $90 \%$ was used to identify all known HPV genotypes.

Anal swabs were collected in a standardized manner with a flocked swab according to the manufacturer's instructions (Eswab, Copan Italia Spa, Brescia, Italy) [24]. The swab was then placed in a sterile tube containing $1 \mathrm{ml}$ of phosphate buffer saline and processed as described above.

Subjects with positive HPV DNA were classified in having a high-risk (HR) HPV, if at least one HR HPV-type was found, or otherwise classified having a low-risk (LR) HPV, if harbouring only low-risk HPV-types. Carcinogenic risk classification was done according to IARC monograph (2007) [25]. Detection was classified in single or multiple HPV if only one or more than one types were characterized. Number of HPV types targeted by nonavalent HPV vaccine were counted in oral and anal samples.

All assays were performed by trained laboratory technicians blinded to clinical information of the patient and to the assay results.

\section{Cytological examination of oral rinses}

Fixed cytological smears were stained according to the Papanicolau method [26] and adequacy evaluated based on the cellularity, as well as on the presence of acceptable fixation and staining.

Two pathologists, blinded to clinical and HPV information, independently evaluated cytological specimens. A cytological assessment of the quality of the obtained cells from each oral sample was undertaken, using standard parameters that include quality of preparation, cellularity, and types of cells present, in addition, data on micro-organisms, leucocytes/inflammatory cells and artefacts were also obtained. Cytological findings were reported using the following categories of the Bethesda 2001 guideline [27] modified as appropriate for the site: NILM (negative for intraepithelial lesion or malignancy), ASC-US (atypical squamous cells of undetermined significance), L-SIL (low grade squamous intraepithelial lesion), or H-SIL (high grade squamous intraepithelial lesion).

\section{Statistical analysis}

Descriptive analysis was conducted to characterize the subjects included in the study. Median values and interquartile ranges (IQR) were used to describe numerical variables, while counts and percentages were employed for qualitative variables. The number of patients with at least one nonavalent vaccine-targeted HPV type over the total number of subjects found to be HPV DNA-positive at each anatomical site were employed to calculate the proportion of vaccine-targeted infections separately for each anatomical site. The association between variables was assessed using chi-squared test or Fisher's exact test, as appropriate. Two-tailed $p$-values were calculated and a value $<0.05$ was considered statistically significant. A univariate logistic regression was used to analyse the strength of association between positive oral HPV detection and other variables, calculating odds ratios (OR) and their 95\% confidence intervals (CI). A multivariable regression analysis, adjusting for variables associated with the outcome at univariate analysis with a $p<0.10$ and forcing age, was carried out. Data management and analysis were performed using SPSS version 23 [28].

\section{Results}

\section{Study population}

The study population included 305 HIV-infected males and general characteristics are shown in Table 1. Briefly, patients were predominantly Caucasians (92.8\%), with a median age of 44.7 years (IQR: 37.4-51.0). Overall, 262 (85.9\%) were MSM. The remaining patients reported HIV acquisition by heterosexual intercourse in 27 cases $(8.9 \%)$ and by intravenous drug use in 4 cases $(1.3 \%)$. Mother-to-child transmission and blood transfusion were registered in 1 case each. In the remaining 10 subjects (3.3\%) HIV transmission modality remained unknown. At anal cancer screening, 289 (94.8\%) patients were on cART since a median of 4.4 years (IQR: 1.99.3). Viroimmunological exams showed HIV RNA below 40 copies/ml in 268 patients $(87.9 \%)$ and a median CD4 cell count of $699 / \mu \mathrm{L}$ (IQR: $529-878$ ). Sixty-two patients (20.3\%) had a CD4 cell count $<500$ cells $/ \mu \mathrm{L}$ and $8<200$ cells $/ \mu \mathrm{L}$. Self-reported previous or current anal/genital condylomatosis was recorded in 112 (36.7\%) men. 
Table 1 Description of the study population $(n=305)$

\begin{tabular}{|c|c|}
\hline \multicolumn{2}{|l|}{ Demographics } \\
\hline Age, median years (IQR) & $44.7(37.4-51.0)$ \\
\hline Caucasian ethnicity, n (\%) & $283(92.8)$ \\
\hline \multicolumn{2}{|l|}{ Clinical, HIV- and cART-related factors } \\
\hline \multicolumn{2}{|l|}{ Main route for HIV acquisition, $\mathrm{n}(\%)$} \\
\hline Homosexual/ Bisexual & $262(85.9)$ \\
\hline Heterosexual & $27(8.9)$ \\
\hline IVDU & $4(1.3)$ \\
\hline Other or unknown & $12(3.9)$ \\
\hline CART, n (\%) & $289(94.8)$ \\
\hline Time on CART, median years (IQR) & $4.4(1.9-9.3)$ \\
\hline \multicolumn{2}{|l|}{ HIV RNA, n (\%) } \\
\hline Not detected & $221(72.5)$ \\
\hline Detectable < 40 copies/ml & $47(15.4)$ \\
\hline$>40$ copies $/ \mathrm{ml}$ & $37(12.1)$ \\
\hline \multicolumn{2}{|l|}{ CD4+ cell count, $n(\%)$} \\
\hline$\geq 500 / \mu l$ & $243(79.7)$ \\
\hline $201-499 / \mu l$ & $54(17.7)$ \\
\hline$\leq 200 / \mu l$ & $8(2.6)$ \\
\hline \multicolumn{2}{|l|}{ Patient-reported health behaviours } \\
\hline \multicolumn{2}{|l|}{ Cigarette smoking, n (\%) } \\
\hline Never & $107(35.1)$ \\
\hline Former & $53(17.4)$ \\
\hline Current & $145(47.5)$ \\
\hline \multicolumn{2}{|l|}{ Use of recreational drug, $\mathrm{n}(\%)$} \\
\hline Never & $224(73.4)$ \\
\hline Former & $34(11.1)$ \\
\hline Current & $47(15.4)$ \\
\hline Current alcohol consumption (>3 units/daily), $\mathrm{n}(\%)$ & $11(3.6)$ \\
\hline \multicolumn{2}{|l|}{ Patient-reported sexual behaviours } \\
\hline \multicolumn{2}{|l|}{ Number of lifetime sexual partners, median (IQR) } \\
\hline Heterosexual males & $10(5.5-35)$ \\
\hline Bi-sexual males & $100(30-300)$ \\
\hline Homosexual males & $100(20-400)$ \\
\hline Receptive oral sex ever, n (\%) & $275(90.2)$ \\
\hline Sexual activity in previous 12 months, $\mathrm{n}(\%)$ & $284(93.1)$ \\
\hline \multicolumn{2}{|l|}{ Type of sexual partner in previous 12 months, $\mathrm{n}(\%)^{\mathrm{a}}$} \\
\hline Heterosexual & $31(10.9)$ \\
\hline Bi-sexual & $44(15.5)$ \\
\hline Homosexual & $209(73.6)$ \\
\hline \multicolumn{2}{|l|}{ Number of partners in previous 12 months, median (IQR) } \\
\hline Heterosexual & $1(1-2)$ \\
\hline Bi-sexual & $7(3-19)$ \\
\hline Homosexual & $4(1-10)$ \\
\hline Previous or current anal condylomatosis, n (\%) & $112(36.7)$ \\
\hline
\end{tabular}

CART: combination antiretroviral therapy; IVDU: intravenous drug use a percentages calculated on 284 sexually active men $(93.1 \%$ of the total) in the last 12 months
Health behaviours assessed in the interview showed that $64.9 \%$ of patients stated cigarette smoke-exposure $(17.4 \%$ previously and $47.5 \%$ currently), $26.5 \%$ reported previous or current recreational drug use, and $3.6 \%$ declared current alcohol intake. Sexual history in the patientreported questionnaire showed at that 152 men (49.8\%) counted more than 100 sexual partners in lifetime. In the previous 12 months, 21 men (6.9\%) declared no sexual activity, while $97(31.8 \%)$ recalled 10 or more partners. The 284 patients who were sexually-active in the preceding 12 months, recalled the following type of sexual partners: only heterosexual in 31 (10.9\%), both sexes in 44 (15.5\%), and only homosexual in 209 (73.6\%) cases. Median number of sexual partners in the previous 12 months were: 1 for heterosexuals (IQR: 1-2), 7 for bisexuals (IQR: 3.018.8), and 4 for homosexuals (IQR: 1-10). Overall, 275 (90.2\%) patients reported receptive oral sex. None of the participating patients had been vaccinated for HPV.

\section{Oral HPV detection}

HPV DNA was found in oral rinses of 64 out of 305 HIVpositive men (20.9\%). HPV genotype was detected by MY09/11 primers in all but 12 cases, in which direct sequencing identified: HPV13 $n=2 ; \operatorname{HPV} 22 n=3 ; \operatorname{HPV} 32 n$ $=8$, HPV97 $n=2 ; \operatorname{HPV} 107 n=1 ; \operatorname{HPV} 145 n=1$.

The upper panel of Fig. 1 shows the different HPV types detected in the oral cavity, according to multiplicity of infection and risk types. Multiple HPV infections were detected in oral rinses in 18 cases (28.1\%): two types in 16 patients, and three to four types in one case each. In HPV DNA-positive oral rinses, alpha-3 species were found in 23 (HPV61 $n=$ 5, HPV62 $n=4$, HPV72 $n=6$, HPV81 and HPV83 in 2 cases each, HPV84 $n=4$ ), alpha-7 in 14 (HPV18, HPV39, HPV45 and HPV59 in 1 case each, HPV70 and HPV85 in 4 cases each, HPV97 $n=2$ ), and alpha9 in 12 cases (HPV16 $n=3$, HPV33 $n=5$, HPV35 and HPV58 in 2 cases each). HPV16 and HPV18 were found in three and one oral samples, respectively. Beta-2 species were detected in 5 samples (HPV22 $n$ = 3, HPV107 and HPV145 in 1 case each). HR types were found in $50 \%$ oral rinses with detectable HPV DNA $(n=32)$, and were more frequently observed in presence of multiple than of single HPV types $(77.8 \%$ versus $39.1 \%$; $p=0.005$ ).

\section{Cytological examination of oral rinses}

Oral rinses samples were not adequate for cytological examination in 27 patients because of low cellularity. Among 278 cases, cytology was normal in 228 (82.0\%) cases, while cellular atypia (ASC-US or more) was found in 50 patients: ASC-US $n=49(17.6 \%)$ and H-SIL $n=1$. In Table 2, the distribution of cytological findings according to HPV risk group is shown. The risk of cellular 


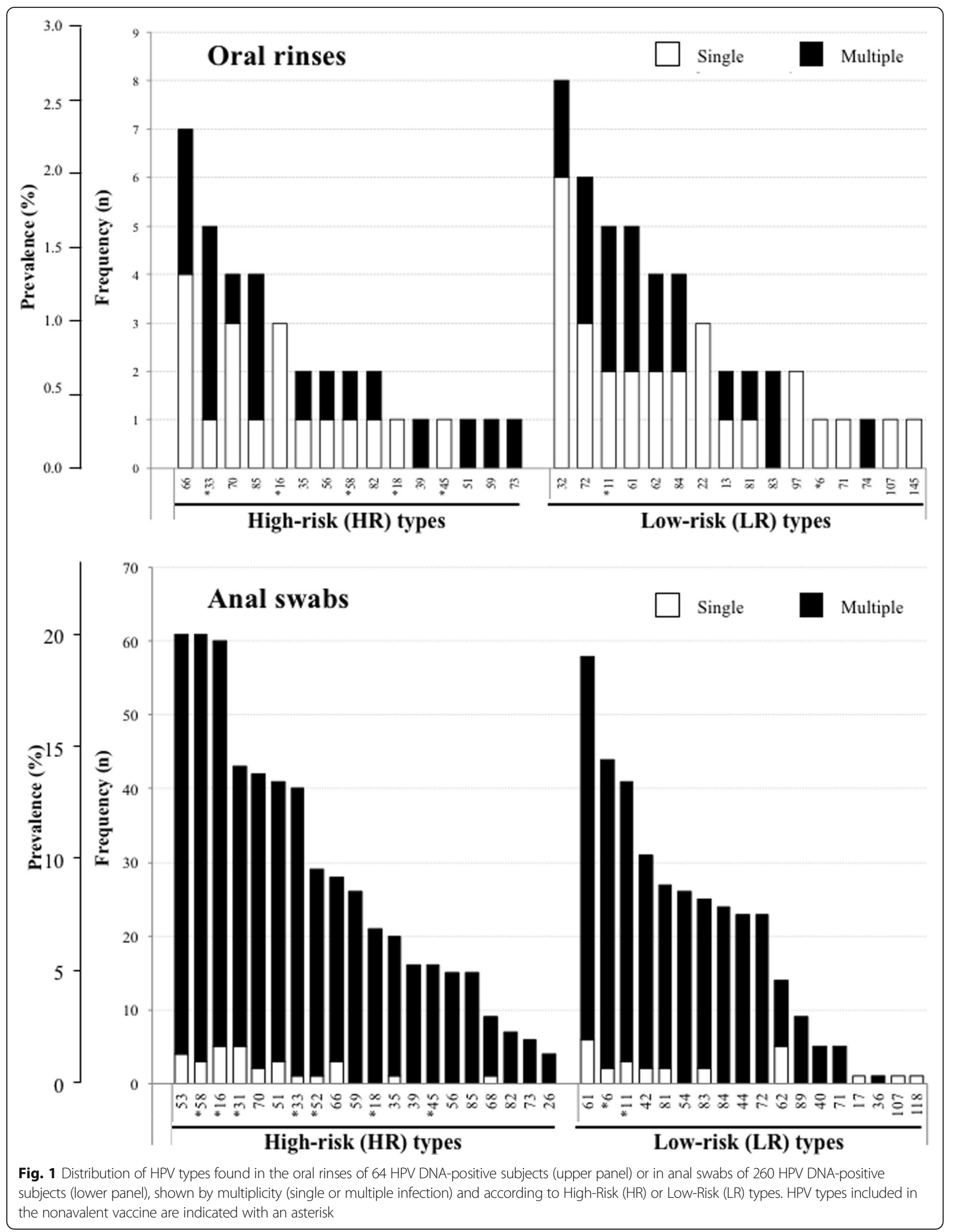


Table 2 Cytological findings in oral rinses of patients with oral HPV infection according to presence of HPV and oncogenic risk

\begin{tabular}{lllll}
\hline & Total & $\begin{array}{l}\text { Negative } \\
\mathrm{n}(\%)\end{array}$ & $\begin{array}{l}\mathrm{HR} \\
\mathrm{n}(\%)\end{array}$ & $\begin{array}{l}\mathrm{LR} \\
\mathrm{n}(\%)\end{array}$ \\
\hline Not adequate & 27 & $26(96.3)$ & $1(3.7)$ & - \\
Negative & 228 & $191(83.8)$ & $15(6.7)$ & $22(9.5)$ \\
ASC-US & 49 & $24(49.0)$ & $15(30.6)$ & $10(20.4)$ \\
H-SIL & 1 & - & $1(100)$ & - \\
Total & 305 & 241 & 32 & 32 \\
\hline
\end{tabular}

LR: HPV infection sustained by only low risk HPV types; HR: HPV infection sustained by at least one high risk HPV type; Negative: negative for intraepithelial lesion or malignancy; ASC-US: atypical squamous cells of undetermined significance; $\mathrm{H}$-SIL: high grade squamous intraepithelial lesion

atypia (ASC-US or more) was higher in presence of HR types when compared to subjects harbouring only LR types (OR 2.31; 95\% CI 0.83-6.69; $p=0.050$ ).

\section{Co-occurrence of HPV DNA at the oral cavity and at the anal site}

HPV DNA was detected at the anal site in 260 out of 305 (85.2\%) male patients. Thus, the proportion of subjects with positive HPV DNA at the oral cavity was significantly lower when compared to that of persons with HPV DNA at the anal site $(21.0 \%$ versus $85.2 \% ; p<$ 0.001). The finding of multiple HPV infection was more frequent in anal $(206 / 260)$ than in oral $(18 / 64)$ samples (79.2\% versus $28.1 \%$; $p<0.001)$. Out of 265 HPV DNApositive patients regardless the anatomic site, only in 59 cases $(22.3 \%)$ HPV DNA was recovered in oral rinses and in anal samples at the same time $(19.3 \%$ of the overall cohort). Detection of HPV at the oral cavity tended to be more frequent in men with HPV DNA detected at anal site when compared to those with negative results (22.7\% versus $11.1 \%, p=0.078)$. Concerning specific HPV types, among the 59 patients with detectable HPV DNA at both anatomical sites, 51 (86.4\%) had completely different HPV types. Out of the remaining eight, seven cases shared only one HPV type (HPV11 $n=2$; HPV61, HPV81 and HPV83 in 1 case each among LR HPV types; HPV51 and HPV66 in 1 case each among HR HPV types), while one patient harboured the same double infection by HR HPV39 and HPV59 types at both sites. In Fig. 1, the distribution of HPV types detected at the anal site are shown by multiplicity and oncogenicity of HPV types.

\section{Variables predictive of positive oral HPV DNA}

Table 3 shows the univariate analysis that found significantly higher odds for oral HPV DNA detection in men who have had more than 5 sexual partners in the previous 12 months (OR 2.72; 95\% CI 1.39-5.34; $p=0.004$ ) or more than 100 partners in lifetime (OR 5.77; 95\% CI $1.31-25.35 ; p=0.020$ ). Further, a significant association between oral HPV DNA and self-reported history of anal/genital condylomatosis (OR 1.86; 95\% CI 1.06-3.24; $p=0.030$ ), use of recreational drugs (OR 2.0; 95\% CI $1.01-3.98, p=0.048)$, and current CD4 cell count below $200 / \mu \mathrm{L}$ when compared to higher values (OR 12.36; $95 \%$ CI 2.43-62.83; $p=0.002$ ) was found.

At multivariable analysis, after adjusting for age as well as all variables found to be associated with positive oral HPV DNA at univariate analysis $(p<0.01)$ and choosing to include "number of sexual partner in the previous 12 months" instead of "number of lifetime partners" to avoid collinearity, a significant independent association between detectable oral HPV DNA and the following conditions was confirmed: CD4 cell count $\leq 200 / \mu \mathrm{L}$ versus $>200 / \mu \mathrm{L} \quad(\mathrm{OR} 12.58 ; 95 \%$ CI 2.19-72.30; $p=0.005)$ and to report $>5$ sexual partners in the previous 12 months versus $0-1$ partner (OR 2.78 ; 95\% CI $1.30-$ 5.93; $p=0.008)$.

\section{Vaccine-targeted HPV types}

A minimum of one nonavalent vaccine-targeted HPV type was found in 17 out of 64 patients (26.6\%) with positive oral HPV DNA, and in 199 out of 260 of patients $(76.5 \%)$ with HPV positivity at the anal site. The nonavalent vaccine-targeted vaccine would have covered all identified HPV types in 11/17 (64.7\%) of patients with HPV at the oral cavity and in 33/199 (16.6\%) of patients with HPV at the anal site (Fig. 1).

\section{Discussion}

In this clinic-based study on Italian HIV-positive men (predominantly MSM) observed within an anal cancer screening, HPV was detected in $20.9 \%$ of subjects at the oral cavity with a significantly lower prevalence than that observed at the anal site. Severe immunodepression and number of sexual partners in the previous 12 months, but not anal HPV detection, were significantly associated with a higher odd of positive oral HPV DNA. Cooccurrence of HPV DNA at the oral cavity and at the anal site was not found, and with few exceptions in patients with infection at both sites HPV types were completely different.

In our study, positive HPV DNA in oral rinses was found in approximately one out of five tested HIVinfected patients, confirming that HPV detection at the oral cavity is quite common in HIV-positive men, especially if MSM. This is in accordance with previous studies [10] and with data from a recently published meta-analysis, that calculated a pooled oral HPV prevalence in MSM with HIV infection of $28.9 \%$ (95\% CI 19.1-38.7) [12]. Further, our results confirm that the prevalence of positive oral HPV DNA in the HIVinfected population in Italy is considerably higher than in young Italian HIV-uninfected adults, where it has 
Table 3 Factors associated with positive oral HPV DNA at univariable and multivariable analysis



$O R$ odds ratio, $a O R$ adjusted odds ratio, $C A R T$ combination antiretroviral therapy

$\S$ In the model all variables found to be associated with positive oral HPV DNA at univariate analysis at $p<0.10$ were included, forcing age. The variable "N. of sexual partner in the previous 12 months" instead of "N. of sexual partners in lifetime" was used to avoid collinearity

${ }^{*} p<0.05 ; \neq p<0.10$ 
been shown around 4\% [29]. Overall, these findings underscore the importance to investigate the oral cavity of HIV-positive men, particularly if MSM, for the presence of HPV to identify patients eventually at higher risk of OSCC development and who might need to undergo cytological examination of oral rinses and stomatological evaluation during follow-up.

With regards to factors predictive of positive oral HPV DNA, our finding of CD4 cell count below $200 / \mu \mathrm{L}$ and higher number of sexual partners in the previous 12 months, agrees with some previous studies [3, 12]. In addition, Beachler et al. [12] reported an increased incidence of oral HPV infection among HIV-infected subjects with decreased CD4 cell count and in those reporting high numbers of partners, whereas male sex, older age and current smoking increased the risk of oral HPV persistence.

In our study, detection of HPV DNA at the oral cavity and at the anal site was not associated to each other. Furthermore, in patients with positive HPV DNA at both anatomical sites, HPV strains were mostly different. The absence of concordance has also been observed in another few studies conducted in HIV-positive men [7, 11, 14, 30, 31]. It is possible that the considerably lower prevalence of HPV DNA at the oral cavity when compared to the anus, together with the lack of concordance in HPV typespecific infections, allows the hypothesis that HPV at the oral site is acquired independently of ano-genital infection or perhaps at different points in time. In fact, selfinoculation by the patient from the ano-genital tract to the oral cavity as transmission route [32], seems unlikely even if considering clearance rates that could differ between the anatomical regions. Nevertheless, one could speculate that, because of differences in local mucosal immunity, non-genital site infections are less likely to reactivate from latency when compared to genital infections, where micro-traumatisms and occurrence of sexually transmitted diseases may decrease the ability to clear anogenital HPV types in respect to oral infections.

In our study, prevalence of most frequent oral HPV types was HPV66 and HPV33 among HR, and HPV32, HPV72, HPV11 and HPV61 among LR. HPV16 was found in only $1.0 \%$ of cases. This finding differed from previous studies, that showed HPV16 as the predominant type [6,33], and may have epidemiological implications. In fact, HPV16 is found in more than $85 \%$ of HPV OSSC [6], and a case-control study estimated that oral HPV16 confers a 50-fold increase in the odds of HPVdriven oro-pharyngeal cancer [31].

In our study population, if the patient had been vaccinated before exposure, the nonavalent vaccine would have targeted at least one HPV strain in only about $27 \%$ patient with detectable HPV DNA at the oral cavity and in $77 \%$ at the anal site. Notably though, nonavalent vaccine would have covered all identified HPV types in more than $60 \%$ of patients with oral HPV DNA, but only less than $20 \%$ of patients with HPV DNA at the anal site. Based on these findings, it may be reasonable to investigate the efficacy of nonavalent vaccines in preventing oral HPV infections.

Our study has some limitations that should be mentioned. Since the tested men have been send at anal cancer screening program by the caring HIV physician, selection bias may have occurred by choosing patients at higher risk of sexually transmitted diseases in general and HPV specifically. Moreover, since a non-standardized interviewer-administered questionnaire was used to collect information regarding sexual history and practices, interview bias may have occurred with distortion of responses by the interviewer or elusion and lies in the answers of the patients. However, our interview was mostly build on fixed-wording questions and multiple-choice answers, reducing the risk of bias.

\section{Conclusions}

In this study on Italian HIV-positive men (predominantly MSM), oral HPV DNA was detected in approximately one fifth of tested men, but prevalence was significantly lower than that observed at the anal site. Low CD4 cell count and increasing number of recent sexual partners significantly increased the odds of positive oral HPV detection. The absence of co-occurrence of HPV DNA at the oral cavity and at the anal site may suggest different routes or timing of infection.

\section{Abbreviations \\ ASC-US: Atypical squamous cells of undetermined significance; \\ CART: combination antiretroviral therapy; HPV: Human Papilloma Virus; HR: Hgh Risk HPV types; H-SIL: High grade squamous intraepithelial lesion; LR: Low risk HPV types; L-SIL: Low grade squamous intraepithelial lesion; NILM: Negative for intraepithelial lesion or malignancy; OSCC: Oro- pharyngeal squamous cell carcinoma}

\section{Acknowledgements}

The authors particularly thank the patients for their participation in the study. Further, they are grateful to the physicians and nurses for valuable collaboration.

\section{Funding}

The study was supported by the SIMIT Scholarship 2015 and by grants from the Italian Ministry of Health (Ricerca Corrente) to National Institute for Infectious Diseases in Rome, Italy.

\section{Availability of data and materials}

The datasets used and/or analysed during the current study are available from the corresponding author on reasonable request.

\section{Authors' contributions}

Each author participated sufficiently in the work giving substantial contributions to realization; $A R G, C S, D L$ performed virological tests; FDN, $A B$ performed cytological examinations; $M G$ collected the oral rinses and anal swabs; ARG, AV, AA2, FL, PP, and CC made contributions to the study conception and design, the acquisition, analysis and interpretation of data, and the drafting and revising of the manuscript. AA1 and MC contributed to the manuscript's revision. All authors contributed to and the intellectual content and gave their final approval to the submitted manuscript. 


\section{Ethics approval and consent to participate}

The protocol conforms to the ethical guidelines of the 1975 Declaration of Helsinki (7th revision, 2013) as reflected in the a priori approval by the "Institutional Review Board" of the National Institute for Infectious Diseases in Rome, Italy (n.42-18/6/2013). A written Informed Consent was obtained from all patients included in the study.

\section{Consent for publication}

Not applicable.

\section{Competing interests}

All authors declare that they have no competing interests.

\section{Publisher's Note}

Springer Nature remains neutral with regard to jurisdictional claims in published maps and institutional affiliations.

\section{Author details}

'HIV/AIDS Unit, INMI "L.Spallanzani", Rome, Italy. ${ }^{2}$ Laboratory of Virology, INMI "L.Spallanzani", Rome, Italy. ${ }^{3}$ Clinical Epidemiology Unit, INMI "L.Spallanzani", Rome, Italy. "Laboratory of Pathology, INMI "L.Spallanzani", Rome, Italy. ${ }^{5}$ National Institute for Infectious Diseases, IRCCS L.Spallanzani, Via Portuense, 292, 00149 Rome, Italy.

\section{Received: 28 June 2017 Accepted: 21 December 2017}

\section{Published online: 08 January 2018}

\section{References}

1. Vandenhende MA, Roussillon C, Henard S, et al. For ANRS EN20 Mortalité 2010 study group. Cancer-related causes of death among HIV-infected patients in France in 2010: evolution since 2000. PLoS One. 2015;10(6): e0129550. https://doi.org/10.1371/journal.pone.0129550.

2. Trickey A, May MT, Vehreschild J, et al. For antiretroviral therapy cohort collaboration (ART-CC). Cause-specific mortality in HIV-positive patients who survived ten years after starting antiretroviral therapy. PLoS One. 2016;11: e0160460. https://doi.org/10.1371/journal.pone.0160460.

3. Beachler DC, Abraham AG, Silverberg MJ, for North American AIDS Cohort Collaboration on Research and Design (NA-ACCORD) of leDEA, et al. Incidence and risk factors of HPV-related and HPV-unrelated head and neck Squamous cell carcinoma in HIV-infected individuals. Oral Oncol. 2014;50: 1169-76. https://doi.org/10.1016/j.oraloncology.2014.09.011.

4. Allam NS, Gandhi Babu DB, et al. Systematic meta-analysis on association of human papilloma virus and oral cancer. Cancer Res Ther. 2016;12:969-74. https://doi.org/10.4103/0973-1482.179098.

5. Schache AG, Powell NG, Cuschieri KS, et al. HPV-related Oropharynx cancer in the United Kingdom: an evolution in the understanding of disease etiology. Cancer Res. 2016;76:6598-606. https://doi.org/10.1158/0008-5472.CAN-16-0633.

6. Kreimer AR, Clifford GM, Boyle P, et al. Human papillomavirus types in head and neck squamous cell carcinomas worldwide: a systematic review. Cancer Epidemiol Biomark Prev. 2005;14:467-75.

7. Parisi SG, Cruciani M, Scaggiante R, et al. Anal and oral human papillomavirus (HPV) infection in HIV-infected subjects in northern Italy: a longitudinal cohort study among men who have sex with men. BMC Infect Dis. 2011;11:150. https://doi.org/10.1186/1471-2334-11-150.

8. Read TRH, Hocking JS, Vodstrcil LA, et al. Oral human papillomavirus in men having sex with men:risk factors and sampling. PLoS One. 2012;7:e49324. https://doi.org/10.1371/journal.pone.0049324.

9. Beachler DC, Sugar EA, Margolick JB, et al. Risk factors for acquisition and clearance of oral human papillomavirus infection among HIV infected and HIV uninfected adults. Am J Epidemiol. 2015;181:40-53. https://doi.org/10. 1093/aje/kwu247.

10. King EM, Oomeer $\mathrm{S}$, Gilson $\mathrm{R}$, et al. Oral human Papillomavirus infection in men who have sex with men: a systematic review and meta-analysis. PLoS One. 2016:1-15. https://doi.org/10.1371/journal.pone.0157976.

11. Ucciferri C, Tamburro M, Falasca K, et al. Prevalence of anal, oral, penile and urethral human Papillomavirus in HIV infected and HIV uninfected men who have sex with men. J Med Virol. 2017; https://doi.org/10.1002/jmv.24943.

12. Prendes BL, Wang SJ, Groppo ER, et al. Oral human papillomavirus infection in men who have sex with men with anal squamous intraepithelial lesions. Head Neck. 2016;38(Suppl 1):E399-405. https://doi.org/10.1002/hed.24006.
13. Wang $L$, Wang $P$, Ren $Y$, et al. Prevalence of high-risk human Papillomavirus (HR-HPV) genotypes and multiple infections in cervical abnormalities from northern Xinjiang, China. PLoS One. 2016;11:e0160698. https://doi.org/10. 1371/journal.pone.0160698.

14. Markowitz LE, Dunne EF, Saraiya M, et al. Human papillomavirus vaccination: recommendations of the advisory committee on immunization practices (ACIP). MMWR Recomm Rep. 2014;63(RR-05):1-30. Erratum in: MMWR Recomm Rep. 2014 Dec 12;63(49):1182

15. Piano Nazionale Prevenzione Vaccinale 2017-2019. http://www.salute.gov.it/ portale/documentazione/p6_2_2_1.jsp?lingua=italiano\&id=2571.

16. Food and Drug Administration. FDA approves Gardasil 9 for prevention of certain cancers caused by five additional types of HPV. Dec.2014. https:/www. fda.gov/BiologicsBloodVaccines/Naccines/ApprovedProducts/ucm426445.htm.

17. Joura EA, Giuliano AR, Iversen $\mathrm{OE}$, et al. A 9-valent HPV vaccine against infection and intraepithelial neoplasia in women. N Engl J Med. 2015;372: $711-23$.

18. European Medicines Agency. Annexe I. Summary of product characteristics. http://www.ema.europa.eu/ema/index.jsp?curl=pages/medicines/human/ medicines/003852/human_med_001863.jsp\&mid=WC0b01ac058001d 124 information/human/003852/WC500189111.pdf.

19. European AIDS Clinical Society. Guidelines, version 9.0. 2017 http://www. eacsociety.org/files/quidelines_8.2-english.pdf.

20. Saiki RK, Scharf S, Faloona F, et al. Enzymatic amplification of beta-globin genomic sequences and restriction site analysis for diagnosis of sickle cell anemia. Science. 1985;230(4732):1350-4. 2999980

21. Garbuglia AR, Piselli P, Lapa D, et al. Frequency and multiplicity of human papillomavirus infection in HIV-1 positive women in Italy. J Clin Virol. 2012; 54:141-6. https://doi.org/10.1016/j.jcv.2012.02.013.

22. Sias C, Garbuglia AR, Piselli P, et al. Comparison of the Abbott RealTime high risk HPV with Genomica HPV clinical Array for the detection of human papillomavirus DNA. APMIS. 2013;121:1054-63. https://doi.org/10.1111/apm.12054.

23. Garbuglia AR, Gentile M, Del Nonno F, et al. An anal cancer screening program for MSM in Italy: prevalence of multiple HPV types and vaccine-targeted infections. J Clin Virol. 2015;72:49-54. https://doi.org/10.1016/j.jcv.2015.09.001.

24. IARC Working Group on the Evaluation of Carcinogenic Risks to Humans. Human papillomaviruses. IARC Monogr Eval Carcinog Risks Hum. 2007;90:1-636.

25. Papanicolaou GN, Traut HF. The diagnostic value of vaginal smears in carcinoma of the uterus. 1941. Arch Pathol Lab Med. 1997;121:211-24.

26. Solomon D, Davey D, Kurman R, et al. The 2001 Bethesda system: terminology for reporting results of cervical cytology. JAMA. 2002;287:2114-9.

27. IBM Corp. Released 2015. IBM SPSS statistics for windows, version 23.0. Armonk NY: IBM Corp.

28. Lupato V, Holzinger D, Höfler D, Menegaldo A, Giorgi Rossi P, Del Mistro A, et al. Prevalence and determinants of oral human Papillomavirus infection in 500 young adults from Italy. PLoS One. 2017;12(1):e0170091. https://doi. org/10.1371/journal.pone.0170091.

29. Videla S, Darwick L, Canadas MP, et al. Natural history of human papillomavirus infections involving anal, penile and oral sites among HIV positive men. Sex Transm Dis. 2013;40:3-10. https://doi.org/10.1097/OLQ. Ob013e31827e87bd.

30. King EM, Gilson R, Beddows S, et al. Oral human papillomavirus (HPV) infection in men who have sex with men: prevalence and lack of anogenital concordance. Sex Transm Infect. 2015;91:284-6. https://doi.org/ 10.1136/sextrans-2014-051955.

31. Taylor $S$, Bunge $E$, Bakker $M$, et al. The incidence, clearance and persistence of non-cervical human papillomavirus infections: a systematic review of the literature. BMC Infect Dis. 2016;16:292. https://doi.org/10.1186/s12879-016-1633-9.

32. Gillison ML, D'Souza G, Westra W, et al. Distinct risk factor profiles for human papillomavirus type 16-positive and human papillomavirus type 16negative head and neck cancers. J Natl Cancer Inst. 2008;100:407-20. https://doi.org/10.1093/jnci/din025.

33. Agalliu I, Wang T, Burk RD. $\beta$ - and $\gamma$-human Papillomavirus types and smoking in head and neck cancer-reply. JAMA Oncol. 2016;2:687-8. https:// doi.org/10.1001/jamaoncol.2016.0973. 